OPEN ACCESS

Edited by:

Ann Dowker,

University of Oxford, United Kingdom

Reviewed by:

Annemie Desoete,

Ghent University, Belgium

Sara Caviola,

University of Cambridge,

United Kingdom

Flávia Heloísa Santos,

Universidade Estadual Paulista Júlio de Mesquita Filho (UNESP), Brazl

${ }^{*}$ Correspondence:

Julia Siemann

julia.siemann@uksh.de,

Julia.siemann@uni-Bremen.de

Specialty section:

This article was submitted to

Developmental Psychology,

a section of the journal

Frontiers in Psychology

Received: 03 August 2017 Accepted: 04 April 2018

Published: 19 April 2018

Citation:

Siemann J and Petermann F (2018) Innate or Acquired? - Disentangling

Number Sense and Early Number Competencies. Front. Psychol. 9:571. doi: 10.3389/fpsyg.2018.00571

\section{Innate or Acquired? - Disentangling Number Sense and Early Number Competencies}

\author{
Julia Siemann ${ }^{* *}$ and Franz Petermann ${ }^{2}$ \\ 1 Department of Medical Psychology and Medical Sociology, University Medical Center Schleswig-Holstein, Kiel, Germany, \\ ${ }^{2}$ Center for Clinical Psychology and Rehabilitation, University of Bremen, Bremen, Germany
}

The clinical profile termed developmental dyscalculia (DD) is a fundamental disability affecting children already prior to arithmetic schooling, but the formal diagnosis is often only made during school years. The manifold associated deficits depend on age, education, developmental stage, and task requirements. Despite a large body of studies, the underlying mechanisms remain dubious. Conflicting findings have stimulated opposing theories, each presenting enough empirical support to remain a possible alternative. A so far unresolved question concerns the debate whether a putative innate number sense is required for successful arithmetic achievement as opposed to a pure reliance on domain-general cognitive factors. Here, we outline that the controversy arises due to ambiguous conceptualizations of the number sense. It is common practice to use early number competence as a proxy for innate magnitude processing, even though it requires knowledge of the number system. Therefore, such findings reflect the degree to which quantity is successfully transferred into symbols rather than informing about quantity representation per se. To solve this issue, we propose a three-factor account and incorporate it into the partly overlapping suggestions in the literature regarding the etiology of different DD profiles. The proposed view on $\mathrm{DD}$ is especially beneficial because it is applicable to more complex theories identifying a conglomerate of deficits as underlying cause of DD.

Keywords: dyscalculia, domain specificity, innate number sense, subtypes, early number competence

\section{SCOPE}

In the present selective review, we discuss normal and abnormal arithmetic development. We present current positions on the central questions of:

(a) precursors for successful mathematical education

(b) risk factors for low math performance

(c) relative contributions of domain-specific and domaingeneral factors

(d) heterogeneity of dyscalculia symptoms.

As a starting point, we will outline the current knowledge on arithmetic acquisition separately for domain-general and domain-specific contributing factors. Based on these findings, we will then explain the key deviations from the regular developmental path present in children 
with dyscalculia according to the literature. For this purpose, typical findings on healthy children with regard to contributions of domain-general as well as domain-specific factors are outlined. Afterward, these are delineated from maladaptive mathematical development.

Next, we will turn to the central question of heterogeneity in developmental dyscalculia (DD). At present, there are still diverse suggested key abnormalities in the literature based on contradictory study results. From this, we will turn to an associated problem: despite the general agreement that there are subtypes of math difficulties, there is an apparent gap with respect to cognitive processes. Here, we wish to put forward that a finer distinction between innate number sense and early number competence helps in disentangling studies contradicting each other. For that purpose, we introduce a three-factor account that is based on past findings and extends previous models. We complement the above by bringing forward several potential reasons leading to different concepts of DD. Finally, we reconcile these seemingly incompatible positions by suggesting how future studies could benefit from our conception of arithmetic development and DD.

\section{HEALTHY MATH DEVELOPMENT: INTERACTIONS BETWEEN DOMAIN-SPECIFIC AND DOMAIN-GENERAL FACTORS}

Before turning to DD and its possible causes, we briefly describe how healthy math development proceeds, because theories on DD are necessarily grounded on this background knowledge. The mammalian brain seems to be equipped with an innate and preverbal ability to differentiate between quantities (e.g., Kucian and von Aster, 2015), the so-called "number sense" (Dehaene and Cohen, 1997). Humans (and other species) can learn to associate this system with symbolic number representations. The latter mechanism apparently evolves in parallel (Hyde, 2011) or hierarchically (von Aster and Shalev, 2007) into the exact and automatic recognition of small amounts of up to four or five items ("subitizing," e.g., Henik et al., 2012; see Piazza, 2010 postulating a precursor object tracking system) and the approximate discrimination between larger quantities ["approximate number system" (ANS), e.g., Feigenson et al., 2004). Similar theories postulate a "one system view" of number representation (Hyde, 2011). Subitizing and ANS thus refer to complementary mechanisms to differentiate small (exact) or large (approximate) numbers, i.e., distinct aspects of the number sense. In concert, they enable the comprehension of cardinality and ordinality (number concept and placement principles, Rapin, 2016). These mathematical principles are crucial for arithmetic and serve as early diagnostic markers (Gray and Reeve, 2014).

Innate basic abilities and acquired general skills both contribute to math development. Geary (2007) discriminates between so-called primary vs. secondary precursors to account for abilities we are biologically endowed with (biologically primary) from skills shaped by environmental influences (biologically secondary). In the following, we will use the more general terms of domain-specific vs. domain-general (e.g., Karmiloff-Smith, 2015). Notably, some studies treat acquired numerical operations (e.g., calculation and arithmetic) as domain-specific (see conceptualization of Gersten and Chard, 1999), and the National Mathematics Advisory Panel even defines number sense as the understanding of the basic concept of numbers (precise representation of small and approximation of large numbers, counting skills, and simple numerical operations; National Mathematics Advisory Panel, 2008) rather than of magnitude per se. This example shows that skills related to early number competencies are taken as proxies for innate number abilities. To disambiguate these distinct concepts (early number competence and magnitude processing), we conceive of number sense as a pre-educational ability (following Berch, 2005) such as magnitude processing and estimation abilities. This differentiation is crucial when interpreting contradictory empirical findings and constitutes the starting point of our threefactor account. For that reason, it is important to consider both contributing factors (primary and secondary), as outlined below for healthy arithmetic development.

\section{Domain-Specific Abilities}

There are several theoretical considerations on math development. For example, von Aster and Shalev (2007) suggest a four-step-model of numerical development from discrete numerosity processing to abstract concepts of magnitude. Therein, domain-specific subitizing is a precursor of counting and subsequently for associating explicit symbolic representations (number words and Arabic digits) with the implicit number sense, culminating in the acquisition of a mental representation of numbers that is spatially organized on a mental number line. The model is based on the triple-code model of number processing (Dehaene et al., 2003) and sketches key brain structures for each developmental stage. Accordingly, there is empirical evidence for brain maturation processes during math learning with regard to structure (Zamarian et al., 2009), function (Rapin, 2016), and connectivity (Moeller et al., 2015). Yet, being explicitly formulated in the context of abnormal mathematical development, the four-step model may not cover the entire spectrum of developmental mechanisms in healthy children. More comprehensive models such as LeFevre et al.'s (2010) three-pathway model commonly schedule three precursors for math development, consisting of domain-specific quantity representation (including subitizing) and domain-general linguistic skills as well as variable indices of spatial processing (see Krajewski and Schneider, 2009; Cirino, 2011, for similar approaches).

These models incorporate the domain-specific number sense in distinct ways. Competing theories suggest either that ANS and acquired mathematical skills depend on common domaingeneral cognitive operations (Park and Brannon, 2014) or that their neuronal representations directly overlap (Lindskog et al., 2014), yet neither accounts for the diverse findings on the relation between ANS and math so far (see Hyde et al., 2016). This may result from the way that number sense and early number 
competence are defined and especially whether ANS is assigned to one (e.g., Szücs and Myers, 2017) or the other (e.g., Jordan et al., 2007).

While von Aster and Shalev (2007) define subitizing as an innate ability that is required for counting (i.e., number sense as we define it here), LeFevre et al.'s (2010) model treats magnitude processing as being synonymous with early numeracy knowledge. Moreover, in empirical studies, there is a tendency to collapse over these competencies (e.g., Powell and Fuchs, 2012). We believe that discrepant findings in the literature are contingent upon these different conceptualizations. Correspondingly, when operationalizing ANS as a proxy for number sense, only moderate levels of correlation with mathematical skills were found in adults (Chen and Li, 2014; Fazio et al., 2014) and in infants (Bonny and Lourenco, 2013) when measured concordantly (cross-sectional studies). Longitudinal studies further point to a genuine causal involvement, as expertise in ANS predicts later math growth (Libertus et al., 2013a). However, this relation decreases with age (Bonny and Lourenco, 2013; Fazio et al., 2014), hinting at a mediating role of the ANS. Thus, Libertus et al. (2013a) found the ANS to work indirectly via early number competencies, which then predict later math achievement. Accordingly, ANS acuity impacts on early number competence but not formal math skills (Libertus et al., 2013b), and the predictive impact of symbolic quantity measures exceeds that of non-symbolic scores (Sasanguie et al., 2012). This may also apply to evidence in the literature that math growth and ANS are apparently uncorrelated (Sasanguie et al., 2013; Szücs et al., 2014). Accordingly, studies operationalizing domain-specific quantity processing via early number competence report a stronger correlation with later mathematical abilities (Jordan et al., 2007; Chu and Geary, 2015). Indeed, Sasanguie et al. (2015) suggest a binary magnitude system with separate modules for exact and approximate quantities. Likewise, Kucian and Kaufmann's (2009) model of number representation for healthy math development explicitly conceptualizes the increasing overlap between different quantity representations with age. The model is in line with the discussed findings as number becomes an abstract concept detached from concrete number representations. Such novel considerations are extensively discussed in a recent metaanalysis taking into account developmental shifts as well as different ANS operationalization measures (Schneider et al., 2017).

In sum, domain-specific magnitude processing (i.e., number sense) is at the heart of most contemporary models seeking to explain developmental trajectories of mathematical processing. Unfortunately, it remains a matter of debate whether magnitude processing is indeed abstract with a dedicated domain-specific module (see the discussion in Cohen Kadosh and Walsh, 2009). Novel conceptualizations of arithmetic development are in need, and existing accounts lack a comprehensive conceptualization that accounts for numerous discrepant findings in the literature (LeFevre, 2016). The matter is further complicated by the diverse influences of secondary precursors that are not easily disambiguated from potential primary causes (see Traeff et al., 2017 on this matter). Moreover, their relative contributions seem to be accompanied by an age-dependent shift. Evidence on domain-general skills will be addressed in the following.

\section{Domain-General Skills}

While the previous paragraph stresses the importance of domainspecific precursors for healthy math development, other studies are devoted to the role of domain-general factors. Several early general skills predict later school math longitudinally, including visuospatial properties (Lauer and Lourenco, 2016; Verdine et al., 2017), intelligence (Dumontheil and Klingberg, 2012; Hornung et al., 2014), linguistic skills (Praet et al., 2013; Zhang et al., 2014), executive control (Bull et al., 2008; Clark et al., 2013), and working memory (LeFevre et al., 2013; Bailey et al., 2014; but see Fuchs et al., 2006). While working memory span has often been considered essential to math skill levels, this seems to be content-specific. In fact, visuospatial rather than verbal WM skills correlate with math achievement in healthy populations (Clearman et al., 2017), whereas patients with DD show stronger correlations with verbal WM (Mammarella et al., 2013). Accordingly, Szücs (2016) identified type of WM impairment (verbal and visuospatial) as contributing to the specific profile of mathematic problems in DD patients. Moreover, the correlation between WM and math may be stronger in children with low number sense capabilities than healthy controls. Therefore, differentiation between control groups and children with DD is essential when examining domain-general factors. Thus, Szücs et al. (2014) found no correlation between WM and math performance in healthy children. A possible explanation is given by the development of an arithmetic fact memory. Healthy individuals may be able to use their number sense to develop early number competencies (i.e., connections between magnitude and numbers, basic arithmetic principles, etc.) as a basis for an arithmetic fact memory. By contrast, children with DD cannot profit from such automated processes, rather relying on immature mental calculation strategies such as counting. These in turn draw heavily on verbal WM capacities (Alloway et al., 2006), probably leading to a stronger connection between arithmetic and WM. Correspondingly, WM seems to be especially important for more sophisticated math operations such as subtraction (Caviola et al., 2014). Finger counting may serve as a compensatory function to offload WM (Crollen et al., 2011) and is frequently observed in DD (Attout and Majerus, 2015). Nonetheless, domain-specific abilities still contribute to later math outcomes over and above general cognitive influences. Thus, elementary and middle school addition both correlate with early number comparison skills irrespective of working memory, visuospatial skills, linguistic performance, and IQ (Bailey et al., 2014). Moreover, early enumeration capacity uniquely accounts for arithmetic achievement when controlling for working memory and executive functions (Gray and Reeve, 2014). A recent meta-analysis further suggests that early number competence but not WM predicts calculation performance in atrisk children (Peng et al., 2016a). In addition, while math training programs were found to have the largest effects on early number competence, improving domain-general cognitive skills does not seem to transfer to enhanced mathematical achievement (see Raghubar and Barnes, 2017). 
Emphasizing the developmental nature of healthy mathematical acquisition may help to reconcile these findings, as both factors (domain-specific and domain-general) apparently contribute to distinct aspects during math growth (Hornung et al., 2014). As a synopsis of the presented considerations, there are many influential factors on arithmetic acquisition: age of participants may determine whether domain-specific or domain-general performance predominantly correlates with math achievement; test format (verbal and visual) may lead to different results especially with respect to correlations with WM; and sample type (healthy and DD) seems to lead to different correlations due to distinct strategies. With this knowledge in mind, the following paragraphs will point to domainspecific and domain-general abnormalities during mathematical development that may cause the disorder labeled DD.

\section{DEVELOPMENTAL DYSCALCULIA}

\section{Nomenclature}

So far, there is no unitary expression for DD, as it is a complex disorder that may be associated with diverse problems including low math performance, low counting skills, weak arithmetic, struggles with calculation, or inabilities in understanding mathematical procedures. Accordingly, synonyms such as "persistent mathematical difficulties" (Morgan et al., 2016), "mathematics learning disability" (Murphy et al., 2007), or "mathematical difficulties" (Schwenk et al., 2017) may be used to delineate profound (maybe innate) magnitude processing from presumably acquired problem with arithmetic (see Morgan et al., 2016). This diversity of expressions for the same basic collection of symptom already indicates that there is neither a unified concept of mathematical disorders nor a consistent etiological explanation thereof. The term "mathematics learning disability" stresses the role of domain-general operations in learning mathematical proficiency and is predominantly applied by opponents of an innate number sense problem (e.g., Rousselle and Noël, 2007). By contrast, "mathematical difficulties" seem to represent a severity-based expression, leaving open the possibility for an innate as well as an acquired etiology.

The current version of the ICD-10 [International Classification of Diseases; World Health Organization [WHO], 1992] classifies dyscalculia among the pervasive and specific developmental disorders (chapter F8) as a specific developmental disorder of scholastic skills (sub-chapter F81) as a mathematical disorder (F81.2) with no further specification. The criteria demand a discrepancy between a child's intelligence level and a standardized math test score as well as adequate mathematical educational circumstances. By contrast, in the latest version of the Diagnostic and Statistical Manual of Mental Disorders (5th ed.; DSM-5; American Psychiatric Association, 2013), DD is listed as specific learning disorder with impairment in mathematics (315.1) and may be grounded on problems with the number sense as well as with arithmetic fact retrieval, calculation, or math reasoning. The release of the revised version ICD-11 is still pending. It is to be expected that similar alterations with respect to intelligence level and mathematical scores will be put forward.
In the following, we will summarize both well-established and more recent positions on pathological math development separately for potential domain-specific and domain-general precursor abilities and integrate the gathered knowledge into a more precise view on DD.

\section{Presumable "Domain-Specific" Symptoms Associated With DD}

Developmental dyscalculia in children is characterized by profound difficulties with various fields of mathematics, including counting principles, transcoding between number digits and number words, comprehension of number syntax, numerical fact knowledge, and fact retrieval (Jordan et al., 2003). A deficient number sense is most frequently related to DD (according to Mazzocco and Thompson, 2005), and correlations between ANS acuity and math proficiency apparently exist prior to mathematical education (Mazzocco et al., 2011; Libertus et al., 2013a) and ANS has a predictive role for math performance in young children (Wong et al., 2017). Analogous to evidence for healthy math development (e.g., Bailey et al., 2014), numerical competence (commonly considered to be domain-specific) of at-risk children uniquely predicts math performance during elementary school even when controlling for domain-general skills (Peng et al., 2016b). By contrast, in adults, DD primarily reflects domain-general fact retrieval deficits and weak phonological processing via an impaired association between both (De Smedt and Boets, 2010).

Immature counting strategies in DD may be causally related to deficient fact knowledge by hindering the build-up of associations between arithmetic operations and solutions (Geary et al., 2012). Similar findings highlight the importance of progressing from procedure-based counting to memory-based fact retrieval (De Visscher and Noël, 2016). Thus, patients with DD are hypersensitive to interference from neighbor problems in multiplication, posing an indirect negative effect by preventing the successful storage of symbol-response associations in longterm memory (De Visscher and Noël, 2016). Still, while children with DD are particularly impaired in grasping the principle of cardinality during counting (Rapin, 2016), which is also predictive of counting in healthy arithmetic development (Moore et al., 2016), the same skill seems to play only a minor role in healthy children. They likewise fail to comprehend this principle despite otherwise healthy mathematical development (Kamawar et al., 2010). Kuhn et al. (2016) infer that DD essentially reflects a deficit of specific precursor abilities that healthy infants are endowed with even before learning arithmetic or calculation (i.e., estimation, enumeration, and transcoding). Longitudinal studies accordingly show that basic quantity-based abilities including number naming, counting, and estimation are stable predictors of arithmetic proficiency during the transition from preschool to kindergarten (VanDerHeyden et al., 2006), elementary school (Methe et al., 2008; Lembke and Foegen, 2009), and high school (Siegler et al., 2012), independent of general intelligence levels (Locuniak and Jordan, 2008). It is therefore likely that quantity processing enables more sophisticated mathematical manipulations. However, a recent meta-analysis 
found symbolic rather than non-symbolic quantity processing measures to be related to low mathematical performance in children with DD (Schwenk et al., 2017). This is in line with the relative contributions of the number sense and of early number competence for healthy arithmetic skills discussed above. The shift from non-symbolic ANS to basic symbolic skills observed in healthy children seems to be aberrant in DD. It is therefore essential to consider supportive skills in contributing to abnormal mathematical development as well. To meet this demand, the following section is devoted to domain-general skills in the context of low mathematical abilities.

\section{Domain-General Deficits in DD}

Apart from poor numerical abilities, low math performance may also be grounded on malfunctioning supportive cognitive operations. Longitudinal and cross-sectional studies repeatedly identified performance differences between children with DD and healthy controls in attention (Ashkenazi et al., 2009; Swanson, 2011), executive functions (especially inhibitory control; Swanson, 2012; Szücs et al., 2013), linguistic skills (as mediator, see Jordan et al., 2015), intelligence (Geary et al., 2008; but see Alloway, 2009), general processing speed (Geary, 2010; Namkung and Fuchs, 2016), and visuospatial processing (Hanich et al., 2001), especially with respect to short-term (Andersson, 2010) or working memory (Ashkenazi et al., 2013; Bugden and Ansari, 2015). Yet evidence on their individual relative contributions is inconclusive, and only few studies systematically explored single cognitive factors in the context of DD (Morgan et al., 2016).

As described initially, diverse skills such as language (Zhang et al., 2014), attention (Morgan et al., 2016), and intelligence (Geary and Moore, 2016) contribute to sophisticated (i.e., healthy) math knowledge. However, findings on healthy mathematical development have limited values for the etiology of DD. Especially with regard to procedural knowledge, evidence on DD is still in need. To our knowledge, only three studies have specifically addressed calculation development (reflecting procedural knowledge) in DD in comparison with healthy controls using longitudinal data so far (according to Peng et al., 2016b). Of these, Alloway (2009) found a correlation between working memory (but not intelligence) and calculation, but without controlling for other variables such as verbal skills or executive functions. In a broader approach, Namkung and Fuchs (2016) found processing speed and attention to predict later calculation expertise in DD, whereas language skills did not explain additional variance. The only study we identified (Peng et al., 2016b) that addressed this matter with data from elementary school (i.e., early math development) suggests that

(a) processing speed (domain-general) and early number competence predict mathematical growth in DD

(b) early number competence mediates the degree to which DD persists at the end of elementary school

(c) children with $\mathrm{DD}$ and comorbid reading disorder compensate their deficits using early number competence rather than domain-general skills.
Peng et al. (2016b) also report independence between linguistic skills and whole number math in DD. In contrast to this finding, others suggest that linguistic deficits may be linked with DD in school-aged children (Fuchs et al., 2006), paralleling the relation between language and healthy math development. Consequently, assuming a direct link between reading disorder and DD - potentially with a causal relationship - is tempting. Yet evidence in this field is contradictory, as longitudinal positive correlations between both clinical samples (Jordan et al., 2002) stand against contrary findings (Andersson, 2010). Moreover, a potentially underlying impact of linguistic deficits on arithmetic fact retrieval in DD (according to Simmons and Singleton, 2008) could not be substantiated unequivocally (e.g., Geary et al., 2012).

This list of deficits associated with DD is far from complete and demonstrates how intricate it is to interpret low math performance in the broader context of mathematical development, especially when compared to healthy children and adult mathematics. This discrepancy stresses the importance of a multifactorial approach in the etiology of DD. In the next sections, we will outline opposing viewpoints in the literature with regard to the question whether abnormal mathematical development is necessarily caused by an underdeveloped number sense and will show how a finer distinction between different precursors for distinct DD subtypes can reconcile ambiguous study results.

\section{Heterogeneity in DD}

In light of the above findings, it appears that the precursors for successful math development differ from those abilities frequently impaired in DD. Healthy math skills are likely to be continuously distributed, whereas DD constitutes profound deficits distinct from the low end of this continuum (see Desoete et al., 2012). Indeed, whereas healthy math performance scores are highly variable from preschool to elementary school (Geary et al., 2000) and persistent interindividual differences only emerge at grade 2 (Jordan et al., 2003), possibly based on changing strategies (Aunola et al., 2013), DD is stable over time (Andersson, 2010). The most obvious demarcation between healthy math development and DD is evident when considering that low initial numerical competence in elementary school is often not clinically significant in follow-up tests anymore (Desoete et al., 2012). Obviously, manifold reasons can account for weak performance in math tests and need to be identified before erroneously diagnosing DD (see Kaufmann et al., 2013 for a discussion). Nonetheless, growth of mathematical proficiency depends decisively on an individual's initial numerical competence even before school (Jordan et al., 2009), suggesting that mathematical cognition may be less unitary than conceptualized in many studies (see Dowker, 2008 on individual differences).

Unfortunately, research so far lacks insight into early developmental influences of deficient precursors specifically in $\mathrm{DD}$, because most studies either address later developmental stages (elementary school) or apply cross-sectional study designs impeding a proper analysis of causal influences (see Peng et al., 2016b). However, children's age represents a major contributing factor to the causes of DD and correlations with other skills. 
Thus, certain precursors are only transiently related to DD (Knievel et al., 2011), and despite the fundamental role of the socalled number sense, domain-general influences must be taken into account to differentiate between DD profiles (Szücs, 2016). For example, domain-general visuospatial (Passolunghi et al., 2008) and decoding skills (Peng et al., 2016b) contribute to arithmetic acquisition but not later math proficiency. Likewise, the predictive role of the ANS decreases with age (Szkudlarek and Brannon, 2017), and while early number competency emerge as an initial predictor, domain-general skills gain more importance through arithmetic development (Geary et al., 2017). Failing to control for such transitory effects may in turn result in contradicting findings such that children with DD can demonstrate age-adequate domain-specific number processing competence. Accordingly, children diagnosed with DD in grade 2 showed comparable number processing profiles compared with a control group in grade 4 (Landerl and Kölle, 2009). Data from Fuchs et al. (2010) suggest that it is crucial to be cautious about the manifest variables chosen to operationalize DD. In that study, only mathematical word problem skills varied with basic numerical abilities, whereas calculation performance did not correlate with other domain-general or domain-specific variables (Fuchs et al., 2010). Neuroimaging findings suggest that the bilateral inferior parietal lobule executes domainspecific magnitude processing (Dehaene et al., 2003) and exhibits disparities in DD (Mórocz et al., 2012). However, the same structure is also engaged in domain-general skills that contribute to arithmetic, like working memory (Dumontheil and Klingberg, 2012), attention (Vandenberghe et al., 2012), and spatial processing (Yang et al., 2012). This emphasizes the diversity of DD profiles and leads to an important question raised in the literature about the etiology of distinct DD subtypes (see Andersson and Östergren, 2012 for a review).

\section{Etiology and Subtypes}

In the last decades of DD research, four distinct classes of theories have emerged (according to Castro-Canizares et al., 2009). The first suggests that a domain-specific number sense deficit underlies DD, either for approximate and analogous quantities (number sense deficit, Wilson and Dehaene, 2007) or for exact and discrete representations thereof (defective number module, Butterworth, 2005a).

Alternatively, DD may stem from poor access to quantity information, i.e., an aberrant communication between brain regions devoted to magnitude and its symbolic representation (access deficit, Rousselle and Noël, 2007).

The third class proposes a generalized magnitude system in the brain (comprising both exact and abstract quantities and extending to numbers, time, and space) that is malfunctioning in persons with DD (a theory of magnitude, Cohen Kadosh et al., 2008).

Finally, a forth class of theories identifies a causal relation between mal-efficient domain-general factors and DD symptoms (cognitive deficits, Geary et al., 2007).

At the interface of these accounts, double deficit theories assume that deficits of multiple neuropsychological abilities contribute to learning disabilities in general
(Wolf and Bowers, 1999). However, there are no consistent findings in the literature. Accordingly, whereas rapid automatized naming of digits and phonological awareness did not predict DD in a previous study (Heikkilä et al., 2016), another study found similar operations (processing speed and verbal comprehension) to correlate with DD symptoms (Willcutt et al., 2013). Moreover, low performance in number comparison tasks is inconclusive with regard to the underlying deficit, because while this problem may stem from a defective innate number processing system (Butterworth, 2005b), an alternative explanation is a deficit in accessing this module (Rousselle and Noël, 2007). Instead, distance and problem size effects may be more informative, as they typically alleviate with development (Holloway and Ansari, 2008) and may be underdeveloped in DD (Skagerlund and Traff, 2016). In addition, there are hardly any physiological findings to support the domain-specific theory on DD (Szücs, 2016). In fact, there is evidence that both domain-general skills and domain-specific abilities represent superordinate predictors of DD (Toll et al., 2016) that are sensitive to training programs (Kuhn and Holling, 2014). Possible reasons may be a potential dependency of number sense performance on WM during early arithmetic development (Vandervert, 2017) or the fact that tests of ANS (representing the number sense) often cannot disentangle perceptual factors drawing on WM from actual numerical skills (Bugden and Ansari, 2015).

The multitude of DD profiles may actually be grounded on separate (and potentially overlapping) etiologies (e.g., Kucian and von Aster, 2015; Skagerlund and Traff, 2016) reflected at first sight in common deficits in arithmetic performance. Thus, the above classes of hypotheses potentially apply to distinct $\mathrm{DD}$ phenotypes and consequently to different underlying causes: whereas a "defective module" (Butterworth, 2005a) or deficient "number sense" (Wilson and Dehaene, 2007) implies that abnormal mathematical development results from an immature magnitude representation; the latter is intact according to the "access deficit" theory (Noël and Rousselle, 2011), which centers on problems retrieving numerosity from symbolic representations (Rousselle and Noël, 2007). Therefore, distinct theories can co-exist and need not be mutually exclusive when more closely investigating the underlying deficits and their operationalization. In the following, we will show that a finer separation of domain-specific deficits dissolves several related issues in DD research.

\section{A Novel Concept of DD Typology}

We suggest that properly characterizing arithmetic development and DD require three factors - as opposed to two in the literature (see Figure 1). Factors 1 and 2 have previously been described. The domain-specific number sense (F1) likely represents the foundation on which arithmetic development rests. During formal math education, various domain-general skills (F2) assist in linking abstract numerosity with symbolic number representations, analogous to a scaffold. The resultant early number competence (F3) comprises tools that are involved in arithmetic operations. 


\section{ARITHMETIC}

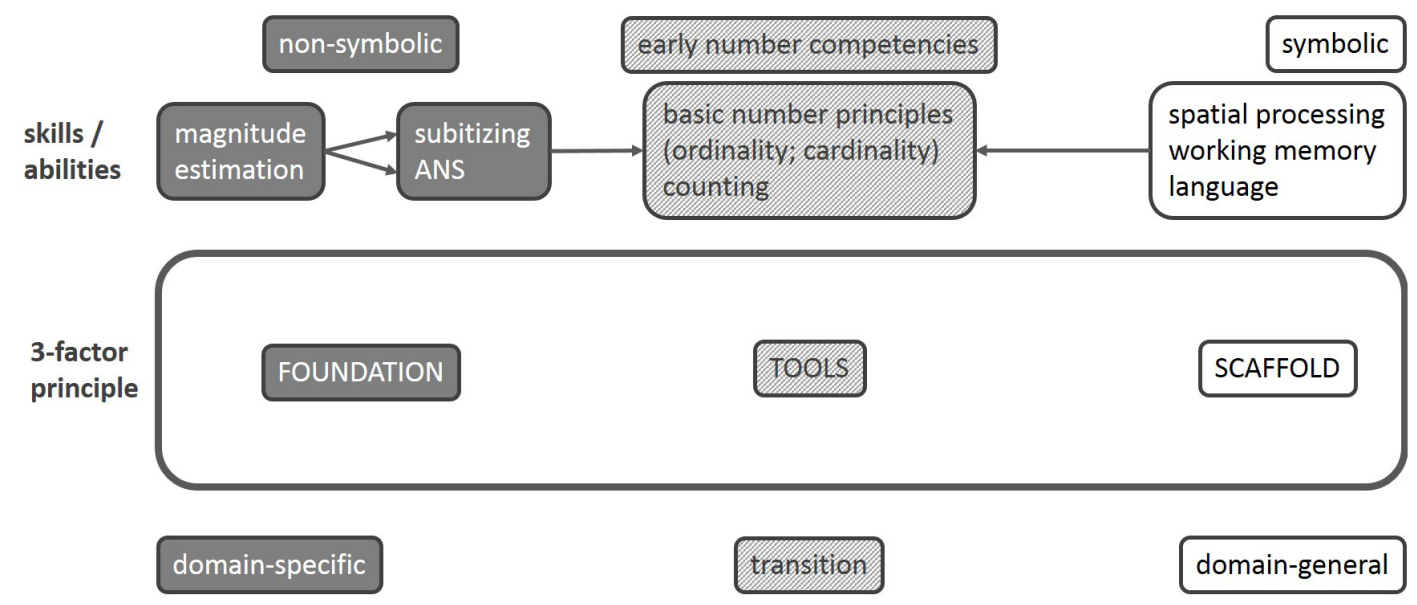

FIGURE 1 | Schematic illustration of the proposed three-factor model. Putative innate skills as well as supporting abilities both contribute to the acquisition of early number competencies required for successful arithmetic.

DD may be caused by different underlying deficits: analogous to a house construction, either the foundation itself is underdeveloped (F1), making it necessary to resort to domaingeneral skills as a scaffold (F2), which is less stable when lacking a proper foundation. But in other cases of $\mathrm{DD}$, there are lowlevel domain-general skills such as working memory: despite an even foundation, an instable scaffold leads to an unsteady house. Third, if the link between non-symbolic and symbolic representations of number (F3) cannot be established, this is analogous to a craftsman using broken tools.

The empirical findings we outlined above are transferable to our three-factor account. Some children with DD show deficits hinting at a poor foundation (magnitude processing; F1), whereas other DD profiles rather accord with low scaffolding support (e.g., working memory or processing speed; F2). While this distinction is well established (Andersson and Östergren, 2012), our view on arithmetic development provides a novel approach to different DD patterns because it highlights the fact that math deficits may be present despite healthy domain-general and domainspecific skills. Crucially, early number competence (F3) is often subsumed under what we consider to be domain-specific skills (F1; as outlined above). By dissociating these two qualitatively different forms of cognition, future studies may succeed in disambiguating DD subtypes. In addition, contradictions between past studies are likely the cause of misconceptions of what a number sense is and what it is not, and distinct correlation patterns between ANS and formal (fact knowledge) compared with informal math (counting) yield empirical support showing a high face validity of this proposed concept (Libertus et al., 2013b). Thus, following Kolkman et al. (2013), domain-specific number sense (F1) primarily assists in establishing a successful mapping between magnitude and symbolic representations, i.e., prior to and during early arithmetic acquisition, for which there is empirical support (Inglis et al., 2011). These considerations are in line with the developmental model of number representation (Kucian and Kaufmann, 2009) introduced previously: while this descriptive model poses a solution for developmental changes observed with increasing arithmetic education, our three-factor account delivers a causal explanation for discrepant findings not only longitudinally (i.e., between different age groups) but also between conceptually different study designs within age groups. That transition appears to rely on domain-general skills (according to Namkung and Fuchs, 2016). Similarly, Hornung et al. (2014) postulate that early number competence in infants results from interacting basic quantity skills with domain-general abilities. We suggest that DD theories centered on domaingeneral deficits are primarily applicable to arithmetic acquisition and may therefore be considered valid especially in accounting for the high variability between age groups both in healthy and in clinical samples (see the discussion in Kaufmann et al., 2013).

Evidence for a third influencing factor (F3) comes from studies hinting at maleficent white matter tracts associated with poor math skills. Both interhemispheric fiber tracts between the IPL (representing the number sense, Cantlon et al., 2011) and intrahemispheric associations between IPL and angular gyrus could be verified (Klein et al., 2013).

Furthermore, the three-factor idea helps reconciling extreme positions of magnitude-based arithmetic vs. direct symbolic activation. The latter is assumed in the "encoding-complex model" (Campbell and Clark, 1988), which neglects domainspecific magnitude representations due to direct activation of numerosity based on parallel relative contributions of number representations. Thus, the foundation of early numerosity (F1) may seemingly become obsolete because studies often test early number competence (number acuity) with symbolic representations (F3). Indeed, recent findings hint at bidirectional correlations between number acuity (F3) and math skills (Lyons 
et al., 2012). While at first sight, this seems to contradict the assumed innateness of number sense processing (F1), it indeed strengthens our stance of a third independent factor of number acuity that was conceptualized as number sense in Lyons et al. (2012).

In addition, our account is sensitive to developmental shifts and therefore provides a high degree of flexibility. Future research should more clearly distinguish between the different concepts in order to differentiate between DD that is based on deficits with early numerosity from innate magnitude problems. Thus, Kaufmann et al. (2013) coined the expression secondary DD for low mathematical skills determined or even caused by low nonnumerical cognitive skills. The matter is further complicated by findings that ANS (as a proxy for magnitude) and schoolbased math are reciprocally related (e.g., Nys et al., 2013; but see Zebian and Ansari, 2012) and that mathematical education impacts on ANS (Piazza et al., 2013; Lindskog et al., 2014). While such results question the assumed innateness of magnitude processing, they likewise provide implications for interventions. If numerosity (in terms of early number competence) turns out to be trainable, low-performing infants should be identified already before formal schooling and participate in specific training programs. Thus far, positive outcomes of such trainings on number acuity (e.g., Nys et al., 2013; Park and Brannon, 2013) could not be established beyond doubt (e.g., Zebian and Ansari, 2012; Obersteiner et al., 2013; see Szücs and Myers, 2017 for a review).

\section{LIMITATIONS}

The articles on DD discussed in the present review are heterogeneous with respect to many aspects impacting on the study results. In the following, we will briefly outline the associated approaches and identify potential strengths and weaknesses:

\section{Design Considerations}

\section{Group Contrasts (Children With/Without DD)}

These studies contrast children with DD and healthy agematched controls with respect to various variables of interest (e.g., mathematical precursors; working memory; and language) and investigate the variance between both samples that each explains. While most efficient in terms of temporal and economic matters, such analyses provide little transferable information (small samples) and no basis to characterize putative causal relationships about developmental trajectories. For these purposes, cross-sectional and longitudinal studies are the means of choice.

\section{Cross-Sectional vs. Longitudinal Studies}

Both study types serve to reveal potential developmental processes. Cross-sectional studies offer a time-economic way to compare different developmental stages with each other but do not enable predictions about causal relations between possible precursor skills and later math achievements, in contrast to longitudinal studies.

\section{Methodological Considerations Choice of Independent Variables}

Another important issue concerns the choice of cognitive functions representing putative precursors and supportive skills for successful arithmetic development. Studies often either investigate domain-specific abilities (Chen and Li, 2014; Fazio et al., 2014) or domain-general skills (LeFevre et al., 2013; Bailey et al., 2014), even though controlling for one factor in the context of another or directly contrasting both (multidimensional approach, see Szücs, 2016) may deliver a more comprehensive insight (Aunola et al., 2004). In addition, studies concentrating on one category (i.e., specific or general) often fail to take into account a sufficient amount of associated factors.

\section{Choice of Dependent Variables}

There is no unitary operationalization of math proficiency or achievement, nor are age and school-based development adequately accounted for. As a result, heterogeneous constructs such as number system knowledge (Sowinski et al., 2015), timed math (Sasanguie et al., 2013), mental calculation(Reeve et al., 2015), standardized math test scores (Chang et al., 2015), or arithmetic fluency (LeFevre et al., 2013) exist for the same overall latent variable termed math proficiency.

\section{Considerations on Sample Criteria Developmental Trajectories}

Irrespective of study design, the classification and comparability of participant sub-groups impact on the associated gain of knowledge. In arithmetic research, longitudinal studies often attend healthy children during the transition from kindergarten to preschool or primary school, thus allowing predictions about regular mathematical proficiency (e.g., VanDerHeyden et al., 2006; Lembke and Foegen, 2009; Siegler et al., 2012). However, the informational value in terms of developmental trajectories of mathematical disorders is limited. Consequently, longitudinal studies on children with low initial number processing abilities (number sense) and potential struggles arising with symbolic representations thereof (transcoding) are more suitable. For this, screening instruments are required that test preschoolers on non-symbolic number processing (e.g., mental number line or non-symbolic quantity estimation tests).

\section{DD Definitions and Diagnostic (Cut-Off) Criteria}

As with math proficiency, the criteria required to sort children into DD (sub)groups are equally inconsistent (see Murphy et al., 2007 for a review). Some studies use the term "persistent mathematical difficulties" to dissociate putative genuine DD from mild and potentially transitional numerical difficulties (Morgan et al., 2016), whereas others collapse over these categories (e.g., Murphy et al., 2007). Furthermore, studies seldom take into account ICD-10 diagnostic criteria for DD, and the associated cut-off criteria are commonly weakened, ranging from the 10th to the 35th percentile (Mazzocco and Thompson, 2005). Thus, qualitative differences between persistent and transient arithmetic weaknesses (Mazzocco and Myers, 2003) impede the comparability between study samples that are based on moderate (e.g., Jordan et al., 2003; Geary, 2004) or low math achievement 
scores (Mazzocco et al., 2011). Besides, reporting comorbid deficits is no established practice, even though these pose additional and fundamental developmental challenges (Szücs, 2016). In addition, concerns have been raised in the past due to the conceptual overlap between mathematical tests with IQ subtests. As a recent advance, the discrepancy criterion was abolished in the United States in 2013 (Schulte-Körne, 2014). DD research mainly relies on convenience samples (often school samples) where standardized IQ indices are not reported at all, or otherwise intelligence level was included as a domain-general regressor. In this respect, weakening the diagnostic criteria as frequently done in DD studies may be advantageous. However, lowering the required discrepancy between participants' math test score and their age-based reference group may be more problematic. Assuming that math skills are dimensionally distributed, this approach may falsely sort healthy low performers into the group of DD patients. This matter is further complicated by the ambiguous definition of DD and potential subgroups.

\section{Considerations on Selected Studies}

The present article should not be misunderstood as a systematic or exhaustive review nor does it make the claim to cover all relevant open questions about DD. The choice of studies is selective and we may not have covered all relevant viewpoints or theories on the related issues. Rather, we wish to point to one essential gap in the approach to research in this field. By drawing attention to the ambiguous conceptualization of "the number sense," we hope to initiate a finer distinction between the discussed abilities/skills in shaping arithmetic sophistication. This may provide new ways of interpreting study results and help reconciling discrepant findings.

In sum, the available studies on DD and math development are confounded with many influential factors. This article served to sensitize researchers in this matter by contrasting evidence and standpoints in the literature from many angles.

\section{REFERENCES}

Alloway, T. P. (2009). Working memory, but not IQ, predicts subsequent learning in children with learning difficulties. Eur. J. Psychol. Assess. 25, 92-98. doi: 10.1027/1015-5759.25.2.92

Alloway, T. P., Gathercole, S. E., and Pickering, S. J. (2006). Verbal and visuospatial short-term and working memory in children: Are they separable? Child Dev. 77, 1698-1716. doi: 10.1111/j.1467-8624.2006.00968.x

American Psychiatric Association (2013). Diagnostic and Statistical Manual of Mental Disorders, 5th Edn. Washington, DC: APA. doi: 10.1176/appi.books. 9780890425596

Andersson, U. (2010). Skill development in different components of arithmetic and basic cognitive functions: Findings from a 3-year longitudinal study of children with different types of learning difficulties. J. Educ. Psychol. 102, 115-134. doi: $10.1037 / \mathrm{a} 0016838$

Andersson, U., and Östergren, R. (2012). Number magnitude processing and basic cognitive functions in children with mathematical learning disabilities. Learn. Individ. Differ. 22, 701-714. doi: 10.1016/j.lindif.2012.05.004

Ashkenazi, S., Black, J. M., Abrams, D. A., Hoeft, F., and Menon, V. (2013). Neurobiological underpinnings of math and reading learning disabilities. J. Learn. Disabil. 46, 549-569. doi: 10.1177/0022219413483174

Ashkenazi, S., Rubinsten, O., and Henik, A. (2009). Attention, automaticity, and developmental dyscalculia. Neuropsychology 23, 535-540. doi: 10.1037/ a 0015347
We complemented these considerations by introducing a novel approach that equally applies to the interpretation of contradictory study results as to the classification of DD subtypes. Thereby, we wished to close this gap and answer some of the questions that follow when looking at individual study results.

\section{OUTLOOK}

In order to differentiate between genuine DD and low math abilities, individual developmental trajectories should be considered in the context of various contributing skills. This idea is pressing given the broad field of domaingeneral and domain-specific precursors that each demonstrates interindividual differences. Disentangling low but healthy math performance from clinically relevant and persistent DD is essential and requires multilevel diagnostic instruments. These in turn depend on the identification of unique precursors of DD that should be screened early on in preschool. For that purpose, future studies are needed that address math development prior to formal mathematical education. As for now, the majority of studies examined school-aged samples, i.e., after having acquired the basic concepts of arithmetic. So far, findings on early number competence (before kindergarten) are still lacking (Morgan et al., 2016). Such studies would help to further disentangle innate abilities (F1) from acquired numerical skills (F3). Furthermore, contradictions between existing studies can possibly be reconciled in a meta-analysis when introducing our three-factor approach.

\section{AUTHOR CONTRIBUTIONS}

JS wrote the preliminary draft. JS and FP revised the manuscript and read and approved the final manuscript.

Attout, L., and Majerus, S. (2015). Working memory deficits in developmental dyscalculia: the importance of serial order. Child Neuropsychol. 21, 432-450. doi: 10.1080/09297049.2014.922170

Aunola, K., Leskinen, E., Lerkkanen, M., and Nurmi, J. (2004). Developmental dynamics of math performance from preschool to grade 2. J. Educ. Psychol. 96, 699-713. doi: 10.1037/0022-0663.96.4.699

Aunola, K., Tolvanen, A., Viljaranta, J., and Nurmi, J. E. (2013). Psychological control in daily parent-child interactions increases children's negative emotions. J. Fam. Psychol. 27, 453-462. doi: 10.1037/a0032891

Bailey, D. H., Siegler, R. S., and Geary, D. C. (2014). Early predictors of middle school fraction knowledge. Dev. Sci. 17, 775-785. doi: 10.1111/desc.12155

Berch, D. B. (2005). Making sense of number sense: implications for children with mathematical disabilities. J. Learn. Disabil. 38, 333-339. doi: 10.1177/ 00222194050380040901

Bonny, J. W., and Lourenco, S. F. (2013). The approximate number system and its relation to early math achievement: Evidence from the preschool years. J. Exp. Child Psychol. 114, 375-388. doi: 10.1016/j.jecp.2012.09.015

Bugden, S., and Ansari, D. (2015). Probing the nature of deficits in the 'Approximate number System'in children with persistent developmental dyscalculia. Dev. Sci. 19, 817-833. doi: 10.1111/desc.12324

Bull, R., Espy, K. A., and Wiebe, S. A. (2008). Short-term memory, working memory, and executive functioning in preschoolers: longitudinal predictors of mathematical achievement at age 7 years. Dev. Neuropsychol. 33, 205-228. doi: $10.1080 / 87565640801982312$ 
Butterworth, B. (2005a). "Developmental dyscalculia," in Handbook of Mathematical Cognition, ed. J. I. D. Campbell (Hove: Psychology Press), 455-467.

Butterworth, B. (2005b). The development of arithmetical abilities. J. Child Psychol. Psychiatry 46, 3-18. doi: 10.1111/j.1469-7610.2004.00374.x

Campbell, J. I., and Clark, J. M. (1988). An encoding-complex view of cognitive number processing: comment on McCloskey, sokol, and goodman. J. Exp. Psychol. Gen. 117, 204-214. doi: 10.1037/0096-3445.117.2.204

Cantlon, J. F., Davis, S. W., Libertus, M. E., Kahane, J., Brannon, E. M., and Pelphrey, K. A. (2011). Inter-parietal white matter development predicts numerical performance in young children. Learn. Individ. Differ. 21, 672-680. doi: 10.1016/j.lindif.2011.09.003

Castro-Canizares, D., Estevez-Perez, N., and Reigosa-Crespo, V. (2009). Contemporary cognitive theories about developmental dyscalculia. Rev. Neurol. 49, 143-148.

Caviola, S., Mammarella, I. C., Lucangeli, D., and Cornoldi, C. (2014). Working memory and domain-specific precursors predicting success in learning written subtraction problems. Learn. Individ. Differ. 36, 92-100. doi: 10.1016/j.lindif. 2014.10.010

Chang, T. T., Rosenberg-Lee, M., Metcalfe, A. W., Chen, T., and Menon, V. (2015). Development of common neural representations for distinct numerical problems. Neuropsychologia 75, 481-495. doi: 10.1016/j.neuropsychologia. 2015.07.005

Chen, Q., and Li, J. (2014). Association between individual differences in nonsymbolic number acuity and math performance: a meta-analysis. Acta Psychol. 148, 163-172. doi: 10.1016/j.actpsy.2014.01.016

Chu, F. W., and Geary, D. C. (2015). Early numerical foundations of young children's mathematical development. J. Exp. Child Psychol. 132, 205-212. doi: 10.1016/j.jecp.2015.01.006

Cirino, P. T. (2011). The interrelationships of mathematical precursors in kindergarten. J. Exp. Child Psychol. 108, 713-733. doi: 10.1016/j.jecp.2010. 11.004

Clark, C. A., Sheffield, T. D., Wiebe, S. A., and Espy, K. A. (2013). Longitudinal associations between executive control and developing mathematical competence in preschool boys and girls. Child Dev. 84, 662-677. doi: $10.1111 /$ j.1467-8624.2012.01854.x

Clearman, J., Klinger, V., and Szücs, D. (2017). Visuospatial and verbal memory in mental arithmetic. Q. J. Exp. Psychol. 70, 1837-1855. doi: 10.1080/17470218. 2016.1209534

Cohen Kadosh, R., Lammertyn, J., and Izard, V. (2008). Are numbers special? an overview of chronometric, neuroimaging, developmental and comparative studies of magnitude representation. Prog. Neurobiol. 84, 132-147. doi: 10.1016/ j.pneurobio.2007.11.001

Cohen Kadosh, R., and Walsh, V. (2009). Numerical representation in the parietal lobes: Abstract or not abstract? Behav. Brain Sci. 32, 313-328. doi: 10.1017/ S0140525X09990938

Crollen, V., Seron, X., and Noël, M.-P. (2011). Is finger-counting necessary for the development of arithmetic abilities? Front. Psychol. 2:242. doi: 10.3389/fpsyg. 2011.00242

De Smedt, B., and Boets, B. (2010). Phonological processing and arithmetic fact retrieval: evidence from developmental dyslexia. Neuropsychologia 48, 3973-3981. doi: 10.1016/j.neuropsychologia.2010.10.018

De Visscher, A., and Noël, M. P. (2016). Similarity interference in learning and retrieving arithmetic facts. Prog. Brain Res. 227, 131-158. doi: 10.1016/bs.pbr. 2016.04.008

Dehaene, S., and Cohen, L. (1997). Cerebral pathways for calculation: double dissociation between rote verbal and quantitative knowledge of arithmetic. Cortex 33, 219-250. doi: 10.1016/S0010-9452(08)70002-9

Dehaene, S., Piazza, M., Pinel, P., and Cohen, L. (2003). Three parietal circuits for number processing. Cogn. Neuropsychol. 20, 487-506. doi: 10.1080/ 02643290244000239

Desoete, A., Ceulemans, A., De Weerdt, F., and Pieters, S. (2012). Can we predict mathematical learning disabilities from symbolic and nonsymbolic comparison tasks in kindergarten? findings from a longitudinal study. Br. J. Educ. Psychol. 82(Pt 1), 64-81. doi: 10.1348/2044-8279. 002002

Dowker, A. (2008). Individual differences in numerical abilities in preschoolers. Dev. Sci. 11, 650-654. doi: 10.1111/j.1467-7687.2008.00713.x
Dumontheil, I., and Klingberg, T. (2012). Brain activity during a visuospatial working memory task predicts arithmetical performance 2 years later. Cereb. Cortex 22, 1078-1085. doi: 10.1093/cercor/bhr175

Fazio, L. K., Bailey, D. H., Thompson, C. A., and Siegler, R. S. (2014). Relations of different types of numerical magnitude representations to each other and to mathematics achievement. J. Exp. Child Psychol. 123, 53-72. doi: 10.1016/j.jecp. 2014.01.013

Feigenson, L., Dehaene, S., and Spelke, E. (2004). Core systems of number. Trends Cogn. Sci. 8, 307-314. doi: 10.1016/j.tics.2004.05.002

Fuchs, L. S., Fuchs, D., Compton, D. L., Powell, S. R., Seethaler, P. M., Capizzi, A. M., et al. (2006). The cognitive correlates of third-grade skill in arithmetic, algorithmic computation, and arithmetic word problems. J. Educ. Psychol. 98, 29-43. doi: 10.1037/0022-0663.98.1.29

Fuchs, L. S., Geary, D. C., Compton, D. L., Fuchs, D., Hamlett, C. L., Seethaler, P. M., et al. (2010). Do different types of school mathematics development depend on different constellations of numerical versus general cognitive abilities? Dev. Psychol. 46, 1731-1746. doi: 10.1037/a0020662

Geary, D. C. (2004). Mathematics and learning disabilities. J. Learn. Disabil. 37, 4-15. doi: 10.1177/00222194040370010201

Geary, D. C. (2007). An evolutionary perspective on learning disability in mathematics. Dev. Neuropsychol. 32, 471-519. doi: 10.1080/ 87565640701360924

Geary, D. C. (2010). "Missouri longitudinal study of mathematical development and disability," in BJEP Monograph Series II, Number 7-Understanding Number Development and Difficulties, (London: British Psychological Society), 31-49. doi: 10.1348/97818543370009X12583699332410

Geary, D. C., Hamson, C. O., and Hoard, M. K. (2000). Numerical and arithmetical cognition: a longitudinal study of process and concept deficits in children with learning disability. J. Exp. Child Psychol. 77, 236-263. doi: 10.1006/jecp.2000. 2561

Geary, D. C., Hoard, M. K., and Bailey, D. H. (2012). Fact retrieval deficits in low achieving children and children with mathematical learning disability. J. Learn. Disabil. 45, 291-307. doi: 10.1177/0022219410392046

Geary, D. C., Hoard, M. K., Byrd-Craven, J., Nugent, L., and Numtee, C. (2007). Cognitive mechanisms underlying achievement deficits in children with mathematical learning disability. Child Dev. 78, 1343-1359. doi: 10.1111/j.14678624.2007.01069.x

Geary, D. C., Hoard, M. K., Nugent, L., and Byrd-Craven, J. (2008). Development of number line representations in children with mathematical learning disability. Dev. Neuropsychol. 33, 277-299. doi: 10.1080/87565640801982361

Geary, D. C., and Moore, A. M. (2016). Cognitive and brain systems underlying early mathematical development. Prog. Brain Res. 227, 75-103. doi: 10.1016/bs. pbr.2016.03.008

Geary, D. C., Nicholas, A., Li, Y., and Sun, J. (2017). Developmental change in the influence of domain-general abilities and domain-specific knowledge on mathematics achievement: an eight-year longitudinal study. J. Educ. Psychol. 109, 680-693. doi: 10.1037/edu0000159

Gersten, R., and Chard, D. (1999). Number sense rethinking arithmetic instruction for students with mathematical disabilities. J. Spec. Educ. 33, 18-28. doi: $10.1177 / 002246699903300102$

Gray, S. A., and Reeve, R. A. (2014). Preschoolers' dot enumeration abilities are markers of their arithmetic competence. PLoS One 9:e94428. doi: 10.1371/ journal.pone.0094428

Hanich, L. B., Jordan, N. C., Kaplan, D., and Dick, J. (2001). Performance across different areas of mathematical cognition in children with learning difficulties. J. Educ. Psychol. 93, 615-626. doi: 10.1037/0022-0663.93. 3.615

Heikkilä, R., Torppa, M., Aro, M., Närhi, V., and Ahonen, T. (2016). Double-deficit hypothesis in a clinical sample: Extension beyond reading. J. Learn. Disabil. 49, 546-560. doi: 10.1177/0022219415572895

Henik, A., Leibovich, T., Naparstek, S., Diesendruck, L., and Rubinsten, O. (2012). Quantities, amounts, and the numerical core system. Front. Hum. Neurosci. 5:186. doi: 10.3389/fnhum.2011.00186

Holloway, I. D., and Ansari, D. (2008). Domain-specific and domain-general changes in children's development of number comparison. Dev. Sci. 11, 644-649. doi: 10.1111/j.1467-7687.2008.00712.x

Hornung, C., Schiltz, C., Brunner, M., and Martin, R. (2014). Predicting first-grade mathematics achievement: The contributions of domain-general cognitive 
abilities, nonverbal number sense, and early number competence. Front. Psychol. 5:272. doi: 10.3389/fpsyg.2014.00272

Hyde, D. C. (2011). Two systems of non-symbolic numerical cognition. Front. Hum. Neurosci. 5:150. doi: 10.3389/fnhum.2011.00150

Hyde, D. C., Berteletti, I., and Mou, Y. (2016). Approximate numerical abilities and mathematics: insight from correlational and experimental training studies. Prog. Brain Res. 227, 335-351. doi: 10.1016/bs.pbr.2016.04.011

Inglis, M., Attridge, N., Batchelor, S., and Gilmore, C. (2011). Non-verbal number acuity correlates with symbolic mathematics achievement: but only in children. Psychon. Bull. Rev. 18, 1222-1229. doi: 10.3758/s13423-011-0154-1

Jordan, J. A., Wylie, J., and Mulhern, G. (2015). Mathematics and reading difficulty subtypes: minor phonological influences on mathematics for 5-7-years-old. Front. Psychol. 6:221. doi: 10.3389/fpsyg.2015.00221

Jordan, N. C., Hanich, L. B., and Kaplan, D. (2003). A longitudinal study of mathematical competencies in children with specific mathematics difficulties versus children with comorbid mathematics and reading difficulties. Child Dev. 74, 834-850. doi: 10.1111/1467-8624.00571

Jordan, N. C., Kaplan, D., and Hanich, L. B. (2002). Achievement growth in children with learning difficulties in mathematics: findings of a two-year longitudinal study. J. Educ. Psychol. 94, 586-597. doi: 10.1037//0022-0663.94. 3.586

Jordan, N. C., Kaplan, D., Locuniak, M. N., and Ramineni, C. (2007). Predicting first-grade math achievement from developmental number sense trajectories. Learn. Disabil. Res. Pract. 22, 36-46. doi: 10.1111/j.1540-5826.2007.00229.x

Jordan, N. C., Kaplan, D., Ramineni, C., and Locuniak, M. N. (2009). Early math matters: kindergarten number competence and later mathematics outcomes. Dev. Psychol. 45, 850-867. doi: 10.1037/a0014939

Kamawar, D., LeFevre, J., Bisanz, J., Fast, L., Skwarchuk, S., Smith-Chant, B., et al. (2010). Knowledge of counting principles: How relevant is order irrelevance? J. Exp. Child Psychol. 105, 138-145. doi: 10.1016/j.jecp.2009.08.004

Karmiloff-Smith, A. (2015). An alternative to domain-general or domain-specific frameworks for theorizing about human evolution and ontogenesis. AIMS Neurosci. 2, 91-104. doi: 10.3934/Neuroscience.2015.2.91

Kaufmann, L., Mazzocco, M. M., Dowker, A., von Aster, M., Gobel, S. M., Grabner, R. H., et al. (2013). Dyscalculia from a developmental and differential perspective. Front. Psychol. 4:516. doi: 10.3389/fpsyg.2013.00516

Klein, E., Moeller, K., and Willmes, K. (2013). A neural disconnection hypothesis on impaired numerical processing. Front. Hum. Neurosci. 7:663. doi: 10.3389/ fnhum.2013.00663

Knievel, J., Petermann, F., and Daseking, M. (2011). Welche Vorläuferdefizite weisen Kinder mit einer kombinierten Rechtschreib- und Rechenschwäche auf? Diagnostica 57, 212-224. doi: 10.1026/0012-1924/a000054

Kolkman, M. E., Kroesbergen, E. H., and Leseman, P. P. (2013). Early numerical development and the role of non-symbolic and symbolic skills. Learn. Instr. 25, 95-103. doi: 10.1016/j.learninstruc.2012.12.001

Krajewski, K., and Schneider, W. (2009). Early development of quantity to number-word linkage as a precursor of mathematical school achievement and mathematical difficulties: findings from a four-year longitudinal study. Learn. Instr. 19, 513-526. doi: 10.1016/j.learninstruc.2008.10.002

Kucian, K., and Kaufmann, L. (2009). A developmental model of number representation. Behav. Brain Sci. 32, 340-341. doi: 10.1017/S0140525X09990069

Kucian, K., and von Aster, M. (2015). Developmental dyscalculia. Eur. J. Pediatr. 174, 1-13. doi: 10.1007/s00431-014-2455-7

Kuhn, J., Ise, E., Raddatz, J., Schwenk, C., and Dobel, C. (2016). Basic numerical processing, calculation, and working memory in children with dyscalculia and/or ADHD symptoms. Z. Kinder Jugendpsychiatr. Psychother. 44, 365-375. doi: 10.1024/1422-4917/a000450

Kuhn, J.-T., and Holling, H. (2014). Number sense or working memory? The effect of two computer-based trainings on mathematical skills in elementary school. Adv. Cogn. Psychol. 10, 59-67. doi: 10.5709/acp-0157-2

Landerl, K., and Kölle, C. (2009). Typical and atypical development of basic numerical skills in elementary school. J. Exp. Child Psychol. 103, 546-565. doi: $10.1016 /$ j.jecp.2008.12.006

Lauer, J. E., and Lourenco, S. F. (2016). Spatial processing in infancy predicts both spatial and mathematical aptitude in childhood. Psychol. Sci. 27, 1291-1298. doi: 10.1177/0956797616655977

LeFevre, J., Berrigan, L., Vendetti, C., Kamawar, D., Bisanz, J., Skwarchuk, S., et al. (2013). The role of executive attention in the acquisition of mathematical skills for children in grades 2 through 4. J. Exp. Child Psychol. 114, 243-261. doi: $10.1016 /$ j.jecp.2012.10.005

LeFevre, J. A. (2016). Numerical cognition: Adding it up. Can. J. Exp. Psychol. 70, 3-11. doi: 10.1037/cep0000062

LeFevre, J. A., Fast, L., Skwarchuk, S. L., Smith-Chant, B. L., Bisanz, J., Kamawar, D., et al. (2010). Pathways to mathematics: Longitudinal predictors of performance. Child Dev. 81, 1753-1767. doi: 10.1111/j.1467-8624.2010.01508.x

Lembke, E., and Foegen, A. (2009). Identifying early numeracy indicators for kindergarten and first-grade students. Learn. Disabil. Res. Pract. 24, 12-20. doi: 10.1111/j.1540-5826.2008.01273.x

Libertus, M. E., Feigenson, L., and Halberda, J. (2013a). Is approximate number precision a stable predictor of math ability? Learn. Individ. Differ. 25, 126-133. doi: 10.1016/j.lindif.2013.02.001

Libertus, M. E., Feigenson, L., and Halberda, J. (2013b). Numerical approximation abilities correlate with and predict informal but not formal mathematics abilities. J. Exp. Child Psychol. 116, 829-838. doi: 10.1016/j.jecp.2013. 08.003

Lindskog, M., Winman, A., and Juslin, P. (2014). The association between higher education and approximate number system acuity. Front. Psychol. 5:462. doi: 10.3389/fpsyg.2014.00462

Locuniak, M. N., and Jordan, N. C. (2008). Using kindergarten number sense to predict calculation fluency in second grade. J. Learn. Disabil. 41, 451-459. doi: $10.1177 / 0022219408321126$

Lyons, I. M., Ansari, D., and Beilock, S. L. (2012). Symbolic estrangement: evidence against a strong association between numerical symbols and the quantities they represent. J. Exp. Psychol. Gen. 141, 635-641. doi: 10.1037/a0027248

Mammarella, I., Caviola, S., Cornoldi, C., and Lucangeli, D. (2013). Mental additions and verbal-domain interference in children with developmental dyscalculia. Res. Dev. Disabil. 34, 2845-2855. doi: 10.1016/j.ridd.2013. 05.044

Mazzocco, M. M., Feigenson, L., and Halberda, J. (2011). Impaired acuity of the approximate number system underlies mathematical learning disability (dyscalculia). Child Dev. 82, 1224-1237. doi: 10.1111/j.1467-8624.2011.01 608. $\mathrm{x}$

Mazzocco, M. M., and Myers, G. F. (2003). Complexities in identifying and defining mathematics learning disability in the primary school-age years. Ann. Dyslexia 53, 218-253. doi: 10.1007/s11881-003-0011-7

Mazzocco, M. M., and Thompson, R. E. (2005). Kindergarten predictors of math learning disability. Learn. Disabil. Res. Pract. 20, 142-155. doi: 10.1111/j.15405826.2005.00129.x

Methe, S. A., Hintze, J. M., and Floyd, R. G. (2008). Validation and decision accuracy of early numeracy skill indicators. Sch. Psychol. Rev. 37, 359-373.

Moeller, K., Willmes, K., and Klein, E. (2015). A review on functional and structural brain connectivity in numerical cognition. Front. Hum. Neurosci. 9:227. doi: 10.3389/fnhum.2015.00227

Moore, A. M., vanMarle, K., and Geary, D. C. (2016). Kindergartners' fluent processing of symbolic numerical magnitude is predicted by their cardinal knowledge and implicit understanding of arithmetic 2 years earlier. J. Exp. Child Psychol. 150, 31-47. doi: 10.1016/j.jecp.2016.05.003

Morgan, P. L., Farkas, G., Hillemeier, M. M., and Maczuga, S. (2016). Who is at risk for persistent mathematics difficulties in the United States? J. Learn. Disabil. 49, 305-319. doi: 10.1177/0022219414553849

Mórocz, I. Á, Janoos, F., van Gelderen, P., Manor, D., Karni, A., Breznitz, Z., et al. (2012). Time-resolved and spatio-temporal analysis of complex cognitive processes and their role in disorders like developmental dyscalculia. Int. J. Imaging Syst. Technol. 22, 81-96. doi: 10.1002/ima.22009

Murphy, M. M., Mazzocco, M. M., Hanich, L. B., and Early, M. C. (2007). Cognitive characteristics of children with mathematics learning disability (MLD) vary as a function of the cutoff criterion used to define MLD. J. Learn. Disabil. 40, 458-478. doi: 10.1177/00222194070400050901

Namkung, J. M., and Fuchs, L. S. (2016). Cognitive predictors of calculations and number line estimation with whole numbers and fractions among at-risk students. J. Educ. Psychol. 108, 214-228. doi: 10.1037/edu0000055

National Mathematics Advisory Panel (2008). Foundations for Success: Final Report of the National Mathematics Advisory Panel. Washington, DC: United States Department of Education.

Noël, M., and Rousselle, L. (2011). Developmental changes in the profiles of dyscalculia: an explanation based on a double exact-and-approximate number 
representation model. Front. Hum. Neurosci. 5:165. doi: 10.3389/fnhum.2011. 00165

Nys, J., Ventura, P., Fernandes, T., Querido, L., Leybaert, J., and Content, A. (2013). Does math education modify the approximate number system? A comparison of schooled and unschooled adults. Trends Neurosci. Educ. 2, 13-22. doi: 10.1016/j.tine.2013.01.001

Obersteiner, A., Reiss, K., and Ufer, S. (2013). How training on exact or approximate mental representations of number can enhance first-grade students' basic number processing and arithmetic skills. Learn. Instr. 23, 125-135. doi: 10.1016/j.learninstruc.2012.08.004

Park, J., and Brannon, E. M. (2013). Training the approximate number system improves math proficiency. Psychol. Sci. 24, 2013-2019. doi: 10.1177/ 0956797613482944

Park, J., and Brannon, E. M. (2014). Improving arithmetic performance with number sense training: an investigation of underlying mechanism. Cognition 133, 188-200. doi: 10.1016/j.cognition.2014.06.011

Passolunghi, M. C., Mammarella, I. C., and Altoe, G. (2008). Cognitive abilities as precursors of the early acquisition of mathematical skills during first through second grades. Dev. Neuropsychol. 33, 229-250. doi: 10.1080/ 87565640801982320

Peng, P., Namkung, J., Barnes, M., and Sun, C. (2016a). A meta-analysis of mathematics and working memory: Moderating effects of working memory domain, type of mathematics skill, and sample characteristics. J. Educ. Psychol. 108, 455-473. doi: 10.1037/edu0000079

Peng, P., Namkung, J. M., Fuchs, D., Fuchs, L. S., Patton, S., Yen, L., et al. (2016b). A longitudinal study on predictors of early calculation development among young children at risk for learning difficulties. J. Exp. Child Psychol. 152, 221-241. doi: 10.1016/j.jecp.2016.07.017

Piazza, M. (2010). Neurocognitive start-up tools for symbolic number representations. Trends Cogn. Sci. 14, 542-551. doi: 10.1016/j.tics.2010.09.008

Piazza, M., Pica, P., Izard, V., Spelke, E. S., and Dehaene, S. (2013). Education enhances the acuity of the nonverbal approximate number system. Psychol. Sci. 24, 1037-1043. doi: 10.1177/0956797612464057

Powell, S. R., and Fuchs, L. S. (2012). Early numerical competencies and students with mathematics difficulty. Focus Except Child 44, 1-16. doi: 10.17161/fec. v44i5.6686

Praet, M., Titeca, D., Ceulemans, A., and Desoete, A. (2013). Language in the prediction of arithmetics in kindergarten and grade 1. Learn. Individ. Differ. 27, 90-96. doi: 10.1016/j.lindif.2013.07.003

Raghubar, K. P., and Barnes, M. A. (2017). Early numeracy skills in preschool-aged children: a review of neurocognitive findings and implications for assessment and intervention. Clin. Neuropsychol. 31, 328-350. doi: 10.1080/13854046.2016. 1259387

Rapin, I. (2016). Dyscalculia and the calculating brain. Pediatr. Neurol. 61, 11-20. doi: 10.1016/j.pediatrneurol.2016.02.007

Reeve, R. A., Paul, J. M., and Butterworth, B. (2015). Longitudinal changes in young children's 0-100 to 0-1000 number-line error signatures. Front. Psychol. 6:647. doi: $10.3389 /$ fpsyg.2015.00647

Rousselle, L., and Noël, M. P. (2007). Basic numerical skills in children with mathematics learning disabilities: a comparison of symbolic vs nonsymbolic number magnitude processing. Cognition 102, 361-395. doi: 10.1016/ j.cognition.2006.01.005

Sasanguie, D., de Smedt, B., and Reynvoet, B. (2015). Evidence for distinct magnitude systems for symbolic and non-symbolic number. Psychol. Res. 81, 231-242. doi: 10.1007/s00426-015-0734-1

Sasanguie, D., Gobel, S. M., Moll, K., Smets, K., and Reynvoet, B. (2013). Approximate number sense, symbolic number processing, or number-space mappings: What underlies mathematics achievement? J. Exp. Child Psychol. 114, 418-431. doi: 10.1016/j.jecp.2012.10.012

Sasanguie, D., Van den Bussche, E., and Reynvoet, B. (2012). Predictors for mathematics achievement? Evidence from a longitudinal study. Mind Brain Educ. 6, 119-128. doi: 10.1111/j.1751-228X.2012.01147.x

Schneider, M., Beeres, K., Coban, L., Merz, S., Schmidt, S. S., Stricker, J., et al. (2017). Associations of non-symbolic and symbolic numerical magnitude processing with mathematical competence: a meta-analysis. Developmental Science 20, e12372. doi: 10.1111/desc.12372

Schulte-Körne, G. (2014). Spezifische Lernstörungen. Z. Kinder Jugendpsychiatr. Psychother. 42, 369-374. doi: 10.1024/1422-4917/a000312
Schwenk, C., Sasanguie, D., Kuhn, J.-T., Kempe, S., Doebler, P., and Holling, H. (2017). (Non)symbolic magnitude processing in mathematically low-achieving and dyscalculic children: a meta-analysis. Res. Dev. Disabil. 64, 152-167. doi: 10.1016/j.ridd.2017.03.003

Siegler, R. S., Duncan, G. J., Davis-Kean, P. E., Duckworth, K., Claessens, A., Engel, M., et al. (2012). Early predictors of high school mathematics achievement. Psychol. Sci. 23, 691-697. doi: 10.1177/0956797612440101

Simmons, F. R., and Singleton, C. (2008). Do weak phonological representations impact on arithmetic development? A review of research into arithmetic and dyslexia. Dyslexia 14, 77-94. doi: 10.1002/dys.341

Skagerlund, K., and Traff, U. (2016). Number processing and heterogeneity of developmental dyscalculia: subtypes with different cognitive profiles and deficits. J. Learn. Disabil. 49, 36-50. doi: 10.1177/0022219414522707

Sowinski, C., LeFevre, J. A., Skwarchuk, S. L., Kamawar, D., Bisanz, J., and Smith-Chant, B. (2015). Refining the quantitative pathway of the pathways to mathematics model. J. Exp. Child Psychol. 131, 73-93. doi: 10.1016/j.jecp.2014. 11.004

Swanson, H. L. (2011). Working memory, attention, and mathematical problem solving: a longitudinal study of elementary school children. J. Educ. Psychol. 103, 821-837. doi: 10.1037/a0025114

Swanson, H. L. (2012). Cognitive profile of adolescents with math disabilities: Are the profiles different from those with reading disabilities? Child Neuropsychol. 18, 125-143. doi: 10.1080/09297049.2011.589377

Szkudlarek, E., and Brannon, E. M. (2017). Does the approximate number system serve as a foundation for symbolic mathematics? Lang. Learn. Dev. 13, 171-190. doi: 10.1080/15475441.2016.1263573

Szücs, D. (2016). Subtypes and comorbidity in mathematical learning disabilities: multidimensional study of verbal and visual memory processes is key to understanding. Prog. Brain Res. 227, 277-304. doi: 10.1016/bs.pbr.2016.04.027

Szücs, D., Devine, A., Soltesz, F., Nobes, A., and Gabriel, F. (2013). Developmental dyscalculia is related to visuo-spatial memory and inhibition impairment. Cortex 49, 2674-2688. doi: 10.1016/j.cortex.2013.06.007

Szücs, D., Devine, A., Soltesz, F., Nobes, A., and Gabriel, F. (2014). Cognitive components of a mathematical processing network in 9-year-old children. Dev. Sci. 17, 506-524. doi: 10.1111/desc.12144

Szücs, D., and Myers, T. (2017). A critical analysis of design, facts, bias and inference in the approximate number system training literature: a systematic review. Trends Neurosci. Educ. 6, 187-203. doi: 10.1016/j.tine.2016. 11.002

Toll, S. W., Kroesbergen, E. H., and Van Luit, J. E. (2016). Visual working memory and number sense: testing the double deficit hypothesis in mathematics. Br. J. Educ. Psychol. 86, 429-445. doi: 10.1111/bjep.12116

Traeff, U., Olsson, L., Östergren, R., and Skagerlund, K. (2017). Heterogeneity of developmental dyscalculia: cases with different deficit profiles. Front. Psychol. 7:2000. doi: 10.3389/fpsyg.2016.02000

Vandenberghe, R., Molenberghs, P., and Gillebert, C. R. (2012). Spatial attention deficits in humans: the critical role of superior compared to inferior parietal lesions. Neuropsychologia 50, 1092-1103. doi: 10.1016/j.neuropsychologia.2011. 12.016

VanDerHeyden, A. M., Broussard, C., and Cooley, A. (2006). Further development of measures of early math performance for preschoolers. J. Sch. Psychol. 44, 533-553. doi: 10.1016/j.jsp.2006.07.003

Vandervert, L. (2017). The origin of mathematics and number sense in the cerebellum: with implications for finger counting and dyscalculia. Cerebellum Ataxias 4:12. doi: 10.1186/s40673-017-0070-x

Verdine, B. N., Golinkoff, R. M., Hirsh-Pasek, K., and Newcombe, N. S. (2017). Vi. discussion and implications: How early spatial skills predict later spatial and mathematical skills. Monogr. Soc. Res. Child Dev. 82, 89-109. doi: 10.1111/ mono. 12285

von Aster, M. G., and Shalev, R. S. (2007). Number development and developmental dyscalculia. Dev. Med. Child Neurol. 49, 868-873. doi: 10.1111/j.1469-8749. 2007.00868.x

Willcutt, E. G., Petrill, S. A., Wu, S., Boada, R., Defries, J. C., Olson, R. K., et al. (2013). Comorbidity between reading disability and math disability: Concurrent psychopathology, functional impairment, and neuropsychological functioning. J. Learn. Disabil. 46, 500-516. doi: 10.1177/0022219413477476

Wilson, A. J., and Dehaene, S. (2007). "Number sense and developmental dyscalculia," in Human Behavior, Learning, and the Developing Brain: Atypical 
Development, eds D. Coch, G. Dawson, and K. W Fischer (New York, NY: Guilford Press), 212-238.

Wolf, M., and Bowers, P. G. (1999). The double-deficit hypothesis for the developmental dyslexias. J. Educ. Psychol. 91, 415-438. doi: 10.1037/0022-0663. 91.3.415

Wong, T. T., Ho, C. S., and Tang, J. (2017). Defective number sense or impaired access? Differential impairments in different subgroups of children with mathematics difficulties. J. Learn. Disabil. 50, 49-61. doi: 10.1177/ 0022219415588851

World Health Organization [WHO] (1992). The ICD-10 Classification of Mental and Behavioural Disorders: Clinical Descriptions and Diagnostic Guidelines. Geneva: World Health Organization.

Yang, J., Han, H., Chui, D., Shen, Y., and Wu, J. (2012). Prominent activation of the intraparietal and somatosensory areas during angle discrimination by intra-active touch. Hum. Brain Mapp. 33, 2957-2970. doi: 10.1002/hbm.21419

Zamarian, L., Ischebeck, A., and Delazer, M. (2009). Neuroscience of learning arithmetic-evidence from brain imaging studies. Neurosci. Biobehav. Rev. 33, 909-925. doi: 10.1016/j.neubiorev.2009.03.005
Zebian, S., and Ansari, D. (2012). Differences between literates and illiterates on symbolic but not nonsymbolic numerical magnitude processing. Psychon. Bull. Rev. 19, 93-100. doi: 10.3758/s13423-011-0175-9

Zhang, X., Koponen, T., Rasanen, P., Aunola, K., Lerkkanen, M. K., and Nurmi, J. E. (2014). Linguistic and spatial skills predict early arithmetic development via counting sequence knowledge. Child Dev. 85, 1091-1107. doi: 10.1111/cdev. 12173

Conflict of Interest Statement: The authors declare that the research was conducted in the absence of any commercial or financial relationships that could be construed as a potential conflict of interest.

Copyright $\odot 2018$ Siemann and Petermann. This is an open-access article distributed under the terms of the Creative Commons Attribution License (CC BY). The use, distribution or reproduction in other forums is permitted, provided the original author(s) and the copyright owner are credited and that the original publication in this journal is cited, in accordance with accepted academic practice. No use, distribution or reproduction is permitted which does not comply with these terms. 